\title{
Synthesis of Polyamides by Direct Polycondensation with Picryl Chloride. II. Reaction Conditions and Mechanism
}

\author{
Hozumi TANAKA, Guo-chuang Wu, Yuki IWANAGA, \\ Kohei SANUI, and Naoya OGATA \\ Department of Chemistry, Sophia University, \\ 7-1 Kioi-cho, Chiyoda-ku, Tokyo 102, Japan
}

(Received March 19, 1982)

\begin{abstract}
A polycondensation reaction of $p$-aminobenzoic acid occurred at room temperature in the presence of picryl chloride to give polyamide in a quantitative yield. Reaction conditions, solvents, temperatures, concentrations of the monomer, and amount of picryl chloride were investigated. A combination of picryl chloride and pyridine was found to be satisfactory for the polycondensation. A model reaction suggests that the polycondensation reaction proceeds through the formation of an active ester from picryl chloride and carboxylic acid, followed by aminolysis with amino group. The polyamide with the solution viscosity of 1.0 was obtained in quantitative yield under optimum conditions. Polyamides from aromatic dicarboxylic acids and aromatic diamine had lower solution viscosities than that from $p$-aminobenzoic acid. The reaction mechanism is discussed.

KEY WORDS Direct Polycondensation / Polyamide / Picryl Chloride / Pyridine / $p$-Aminobenzoic Acid /
\end{abstract}

Polycondensation reactions are generally carried out at elevated temperatures above $200^{\circ} \mathrm{C}$ in order to eliminate volatile products, such as water or alcohol, and thereby shift the equilibrium towards the formation of polymer. On the other hand, polycondensation can be enhanced by using active monomers such as acid chlorides ${ }^{1-3}$ or activated esters $^{4-6}$ so that polycondensation reactions take place in solution under mild conditions.

It was reported in the previous paper ${ }^{7}$ that the polyamides and polyesters were formed with picryl chloride under mild conditions.

Various polyamides were prepared by this synthetic method as shown below, using picryl chloride as a condensation agent:
This paper describes the results of the polycondensation carried out to establish optimum reaction conditions for the formation of the polyamide from $p$-aminobenzoic acid by a direct polycondensation reaction using picryl chloride, and this reaction mechanism is also discussed.

\section{EXPERIMENTAL}

Picryl chloride and other aromatic halocompounds of special grade purity were used as purchased. Solvents were purified by conventional procedures. The general procedure used for the synthesis of the polyamide from $p$-aminobenzoic acid is as follows:

$$
\begin{aligned}
& \mathrm{H}_{2} \mathrm{~N}-\mathrm{R}-\mathrm{NH}_{2}+\mathrm{HOOC}-\mathrm{R}^{\prime}-\mathrm{COOH}+\mathrm{O}_{2}-\mathrm{NO}_{2}^{\mathrm{N}-\mathrm{Cl}_{2}} \\
& \underset{\text { in pyridine }}{\longrightarrow}+\mathrm{NH}-\mathrm{R}-\mathrm{NHCO}-\mathrm{R}^{\prime}-\mathrm{COt}+\mathrm{Py} \cdot \mathrm{HCl}+\mathrm{O}_{2}
\end{aligned}
$$


$0.004 \mathrm{~mol}$ of $p$-aminobenzoic acid was dissolved in a mixed solvent of $5 \mathrm{~cm}^{3}$ of pyridine and $5 \mathrm{~cm}^{3}$ of $\mathrm{N}$-methyl-2-pyrrolidone (NMP), and then 0.005 mol of picryl chloride was added to the solution with stirring at room temperature.

As soon as the picryl chloride was added, an exothermic reaction took place and the solution became heterogeneous with the separation of a solid polymer. When lithium chloride was added to NMP, the reaction proceeded in a homogeneous phase.

After a given period of time, excess acetone was added to remove the solvent and by-products from the polymer which was then isolated by filtration. The polymer was repeatedly washed with water, dilute aqueous sodium hydroxide and then water, followed by acetone, and dried under reduced pressure. The solution viscosities of the polyamides were determined in concentrated sulfuric acid at $30^{\circ} \mathrm{C}$. The model reaction was carried out by benzoic acid or pivaric acid in the presence of picryl chloride and pyridine in dioxane, and the resulting compounds were identified by infrared spectroscopic analysis.

\section{RESULTS AND DISCUSSION}

Table I summarizes the results of synthesis of the polyamides from various monomers by direct polycondensation in the presence of picryl chloride. This table shows that polyamides were obtained in good yield from $p$-aminobenzoic acid (4-ABA), while the combination of dicarboxylic acid and diamine failed to give satisfactory results in terms of polymer yield and solution viscosity.

When triethylamine was used in place of pyridine, no polymer was obtained.

The polycondensation of aliphatic dicarboxylic acid and diamine resulted in a higher yield than that of $\omega$-amino acid such as $\varepsilon$-aminocaproic acid. When $\mathrm{LiCl}$ was added in a mixed solvent of NMP and pyridine, both the yield and solution viscosity of the resulting polyamide increased compared to the results obtained in the absence of $\mathrm{LiCl}$. Table II shows the results of solvent effect on the polycondensation of 4-ABA in the presence of lithium chloride. An inferior result of the polyamide was obtained in nonpolar solvents such as benzene compared to the results in aprotic solvents such as $N$-methyl-2pyrrolidone (NMP). The reason for this may be that poly(4-ABA) could not dissolve in nonpolar solvents.

On the other hand, aprotic solvents such as NMP, tetramethylurea (TMU), or dimethylacetamide (DMAc), yielded relatively higher molecular weight polyamides, presumably because the growth

Table I. Synthesis of polyamide with picryl chloride at room temperature

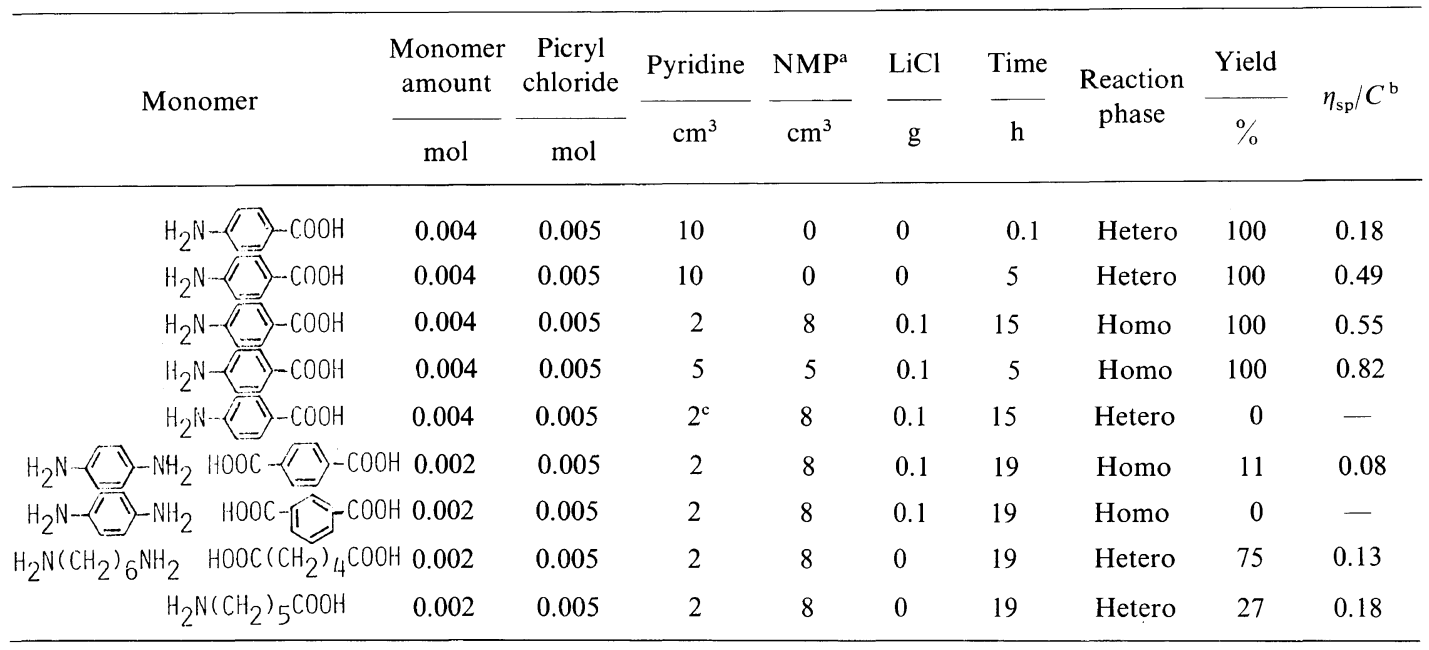

a $N$-Methyl-2-pyrrolidone.

b $0.1 \mathrm{~g} / 10 \mathrm{~cm}^{3}$ in concd $\mathrm{H}_{2} \mathrm{SO}_{4}$ at $30^{\circ} \mathrm{C}$.

c Triethylamine. 
reaction might continue owing to the swelling of the poly(4-ABA) in these solvents. No polymer was obtained in dimethylformamide (DMF) and dimethyl sulfoxide (DMSO) where some side reactions may have participated owing to the presence of the oxidation reagent.

Figure 1 shows the time conversion curve for the polycondensation reaction of 4-ABA with picryl chloride in a mixed solvent of NMP and pyridine $(1 / 1)$.

The polycondensation of 4-ABA took place very rapidly in a mixed solvent of NMP and pyridine in the presence of $1.5 \mathrm{wt} \%$ of $\mathrm{LiCl}$. The solution viscosity of the polymer reached a constant value within $1 \mathrm{hr}$ and the growing reaction was rapid even at room temperature.

Figure 2 summarizes the dependence of the solution viscosities of the resulting polyamides on the reaction temperature. The reaction temperature influenced the solution viscosity and the value showed a maximum at $20-30^{\circ} \mathrm{C}$ as can be seen in Figure 3 . The polycondensation above $40^{\circ} \mathrm{C}$ gave polyamides having low solution viscosities, presumably owing to thermal degradation of the reaction intermediate

Table II. Solvent effect on the polycondensation of 4-aminobenzoic acid

\begin{tabular}{|c|c|c|c|c|}
\hline \multirow{2}{*}{ Solvent } & \multirow{2}{*}{$\begin{array}{l}\text { Dielectric } \\
\text { constants }\end{array}$} & \multirow{2}{*}{$\begin{array}{c}\text { Reaction } \\
\text { phase }\end{array}$} & \multicolumn{2}{|c|}{ Polymer } \\
\hline & & & Yield $/ \%$ & $\eta_{\mathrm{sp}} / C^{\mathrm{b}}$ \\
\hline Benzene & 2.27 & Hetero & 63 & 0.25 \\
\hline Chloroform & 4.20 & Hetero & 100 & 0.12 \\
\hline Tetrahydrofuran & 7.39 & Hetero & 100 & 0.20 \\
\hline Tetramethylurea & 23.1 & Homo & 100 & 0.79 \\
\hline$N$-Methyl-2-pyrrolidone & 32.2 & Homo & 100 & 0.87 \\
\hline Hexamethylphosphoric triamide & 34.0 & Homo & 62 & 0.12 \\
\hline Dimethylformamide & 36.6 & Homo & 0 & - \\
\hline Dimethylacetamide & 37.8 & Homo & 100 & 0.64 \\
\hline Dimethyl sulfoxide & 48.9 & Homo & 0 & - \\
\hline
\end{tabular}

a Reaction condition: monomer, $0.004 \mathrm{~mol}$, pyridine, $5 \mathrm{~cm}^{3}$, solvents, $5 \mathrm{~cm}^{3}$, in the presence of $\mathrm{LiCl}$, for $2 \mathrm{~h}$, at room temperature.

b $0.1 \mathrm{~g} / 10 \mathrm{~cm}^{3}$ in concd $\mathrm{H}_{2} \mathrm{SO}_{4}$ at $30^{\circ} \mathrm{C}$.

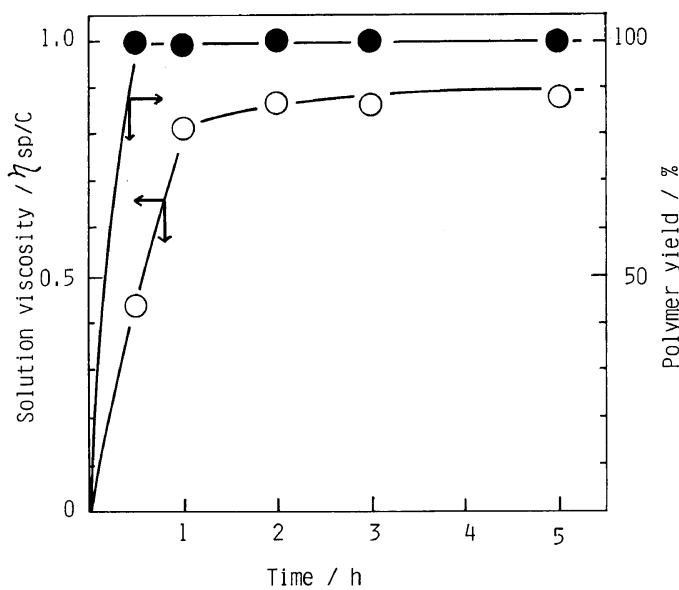

Figure 1. Rate of polycondensation of 4-aminobenzoic acid at room temperature. Reaction condition: monomer concn. $0.004 \mathrm{~mol}$ in $10 \mathrm{~cm}^{3}$ of a mixed solvent of pyridine and NMP.

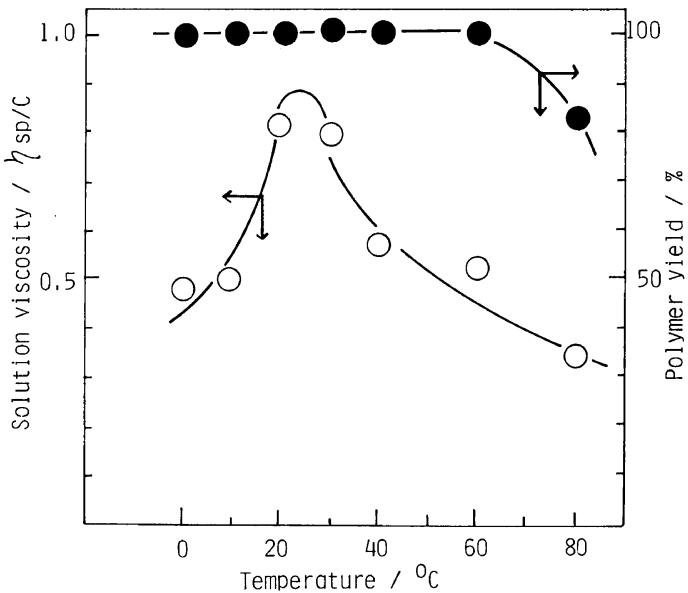

Figure 2. Effect of reaction temperature on the polycondensation of 4-aminobenzoic acid. Reaction condition: monomer concn. $0.004 \mathrm{~mol}$ in $10 \mathrm{~cm}^{3}$ of a mixed solvent of pyridine and NMP, time, $1 \mathrm{~h}$. 


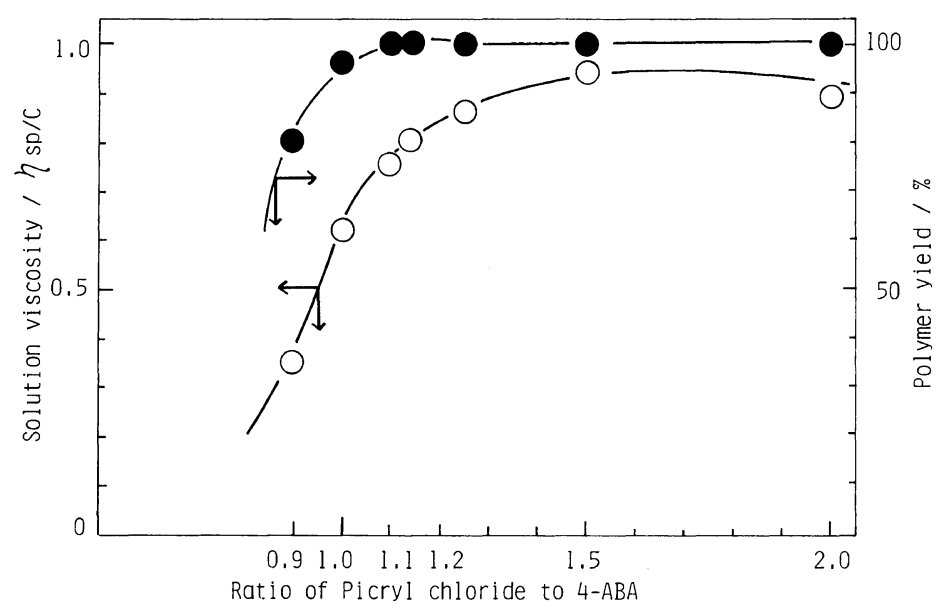

Figure 3. Effect of the amount of picryl chloride on the polycondensation of 4-aminobenzoic acid at room temperature. Reaction condition: monomer concn. $0.004 \mathrm{~mol}$ in $10 \mathrm{~cm}^{3}$ of a mixed solvent of pyridine and NMP, time, $1 \mathrm{~h}$.

which was possibly enhanced at higher temperatures.

Figure 3 indicates the effect of the amount of picryl chloride to the monomer on the polycondensation of 4-ABA. The solution viscosities of the resulting polymers increased with an increase in the amount of picryl chloride, reaching a value of 0.9 . The optimum molar ratio of picryl chloride to 4-ABA was found to be $1.5: 1$. When an excess amount of picryl chloride was added, the separation of the polymer from the reaction mixture was very difficult because of by-products such as pyridinium salt from pyridine with picryl chloride.

Figure 4 shows the effect of monomer concentration on the solution viscosities of poly(4-ABA). The monomer concentration was found to have a significant influence on the solution viscosity of poly(4-ABA). When the polycondensation reaction of 4-ABA was carried out at a concentration between $0.2-0.4 \mathrm{~mol} \mathrm{dm}^{-3}$, the resulting polyamide had a solution viscosity of $c a$. 0.8 , while at a monomer concentration of more than $0.6 \mathrm{~mol}$ $\mathrm{dm}^{-3}$, the resulting polyamide had a low solution viscosity.

Acid acceptors were also an important factor in obtaining polyamides of high solution viscosity. Therefore, various basic solvents were used both as solvents and as acid acceptors in the polycondensation of 4-ABA in a mixed solvent of NMP. As shown in Table III, pyridine was a specific

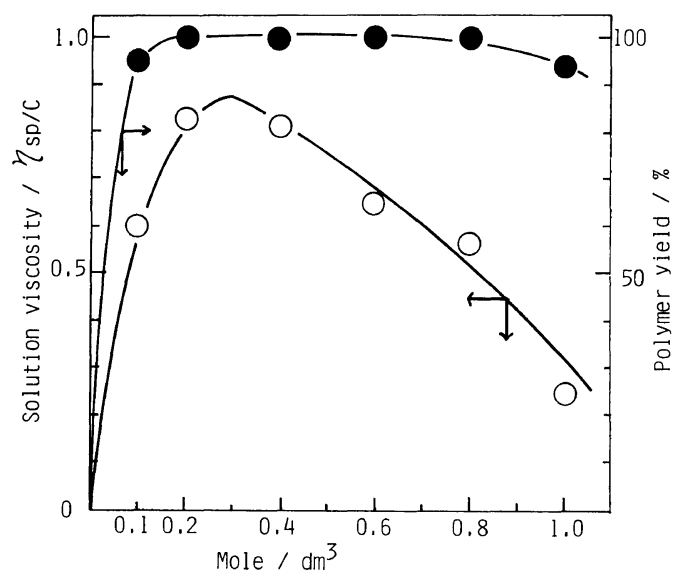

Figure 4. Effect of monomer concentration of 4aminobenzoic acid on polycondensation reaction at room temperature. Reaction condition: monomer concn. $0.004 \mathrm{~mol}$ in $10 \mathrm{~cm}^{3}$ of a mixed solvent of pyridine and NMP for $1 \mathrm{~h}$.

solvent to yield polyamides having high solution viscosity. The yield and solution viscosity of the resulting polyamides might be related to the basicity and the steric hindrance of the substituent of pyridine derivatives at the stage when a reaction intermediate was formed.

Figure 5 shows the effect of pyridine content in mixed solvents on the polycondensation of 4-ABA. NMP containing $70 \%$ pyridine as a solvent yielded 
Table III. Effect of base-solvent on the polycondensation of 4-aminobenzoic acid

\begin{tabular}{lcccc}
\hline \multicolumn{1}{c}{ Solvent } & $\mathrm{p} K_{\mathrm{a}}$ & Polymer & \multicolumn{2}{c}{ Polymer } \\
\cline { 4 - 5 } & & phase & Yield $/ \%$ & - \\
\hline 2-Chloropyridine & 0.49 & Homo & 0 & - \\
Quinoline & 4.81 & Homo & trace & 0.85 \\
Pyridine & 5.25 & Homo & 100 & $-C^{\mathrm{b}}$ \\
3-Picoline & 5.63 & Homo & 100 & 0.72 \\
2-Picoline & 5.94 & Homo & 58 & -13 \\
4-Picoline & 6.03 & Homo & trace & - \\
2,6-Lutidine & 6.60 & Homo & trace & - \\
Triethylamine & 10.75 & Hetero & & \\
\hline
\end{tabular}

a Polycondensation conditions: monomer, $0.004 \mathrm{~mol}$, in NMP, $5 \mathrm{~cm}^{3}$, base-solvents, $5 \mathrm{~cm}^{3}$ in the presence of $\mathrm{LiCl}$, for $14 \mathrm{~h}$, at room temperature.

b $0.1 \mathrm{~g} / 10 \mathrm{~cm}^{3}$ in concd $\mathrm{H}_{2} \mathrm{SO}_{4}$ at $30^{\circ} \mathrm{C}$.

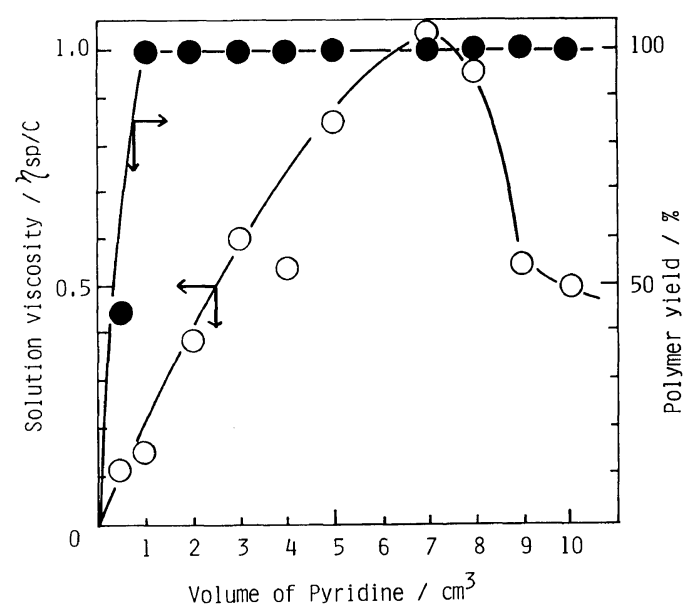

Figure 5. Effect of volume of pyridine on direct polycondensation of 4-aminobenzoic acid in a mixed solvent of pyridine and $\mathrm{N}$-methyl-2-pyrrolidone. Reaction condition: monomer concn. $0.004 \mathrm{~mol}$ in $10 \mathrm{~cm}^{3}$ of a mixed solvent at room temperature.

a polyamide with a maximum solution viscosity as high as 1.0. The component in the mixed solvent might be favorable to better solubility of poly(4ABA), which may enhance the growth reaction of the polymer chains.

Results of the polycondensation of 4-ABA in the presence of various aromatic halo-compounds as a condensation agent are summarized in Table IV. No polymer was obtained when 2,4-dinitrofluorobenzene and 2,4-dinitrochlorobenzene were used in place of picryl chloride. Thus, picryl chloride had good reactivity for initiating direct polycondensation in the synthesis of polyamides.

\section{REACTION MECHANISM}

As described previously, pyridine played a unique role not only as a solvent but also as an acid acceptor for the reaction. In functioning as an acid acceptor amount of pyridine required for the reaction would be twice the number of moles of the monomer. However, only a low yield of polyamide was obtained from 4-ABA under this condition. Thus, it seems possible that pyridine participates in the reaction in addition to its roles as a solvent and an acid acceptor. It is known ${ }^{8}$ that a pyridinium salt is formed between picryl chloride and pyridine as shown below:

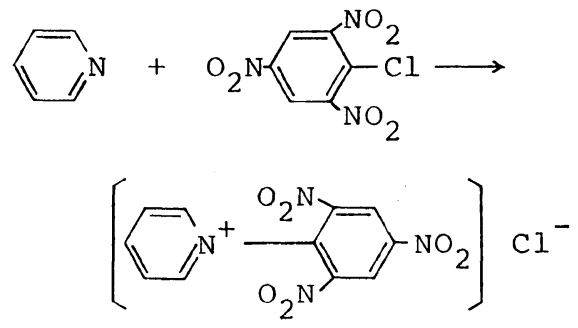

This pyridinium salt reacts with carboxylic acid in the following scheme to form either acid chloride or active ester.

It was reported ${ }^{9}$ that the reaction of 2-fluoro1,3,5-trinitrobenzene (FTNB) with cinnamic acid gave cinnamoyl fluoride in $68 \%$ yield in the presence 
Table IV. Effect of various aromatic halo-compounds on polycondensation reaction of 4-aminobenzoic acid ${ }^{\mathrm{a}}$

\begin{tabular}{|c|c|c|c|c|c|c|}
\hline \multirow{2}{*}{$\begin{array}{c}\text { Aromatic } \\
\text { halo-compound }\end{array}$} & \multirow{2}{*}{$\frac{\text { Pyridine }}{\mathrm{cm}^{3}}$} & \multirow{2}{*}{$\frac{\mathrm{NMP}^{\mathrm{b}}}{\mathrm{cm}^{3}}$} & \multirow{2}{*}{$\frac{\text { Time }}{\mathrm{h}}$} & \multirow{2}{*}{$\begin{array}{l}\text { Reaction } \\
\text { phase }\end{array}$} & \multicolumn{2}{|c|}{ Polymer } \\
\hline & & & & & Yield $/ \%$ & $\eta_{\mathrm{sp}} / C^{\mathrm{c}}$ \\
\hline $\mathrm{O}_{2} \mathrm{~N}-\mathrm{C}$ & 5 & 5 & 23 & Homo & trace & - \\
\hline $\mathrm{O}_{2} \mathrm{~N}-$ & 5 & 5 & 18 & Homo & 0 & - \\
\hline $\mathrm{O}_{2} \mathrm{~N}-\mathrm{C}$ & 5 & 5 & 18 & Homo & 100 & 0.85 \\
\hline
\end{tabular}

a Polycondensation condition: monomer, $0.004 \mathrm{~mol}$, in the presence of $\mathrm{LiCl}$, at room temperature.

b $N$-Methyl-2-pyrrolidone.

c $0.1 \mathrm{~g} / 10 \mathrm{~cm}^{3}$ in concd $\mathrm{H}_{2} \mathrm{SO}_{4}$ at $30^{\circ} \mathrm{C}$.

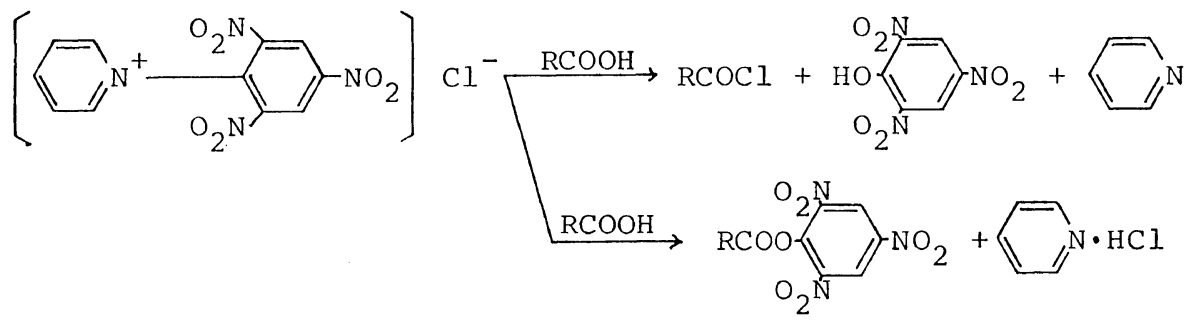

of triethylamine in acetonitrile, and that the reaction of 1-fluoro-2,4-dinitrobezene with phenylacetic acid gave 2,4-dinitrophenyl phenylacetate in a $71 \%$ yield by the same method. Therefore, the model reaction was carried out with picryl chloride and pivaric acid or benzoic acid in pyridine or in dioxane in the presence of pyridine. Results of the model reaction are shown in Figure 6, where the reaction of picryl chloride with pivaric acid leads to the formation 2,4,6-trinitrophenyl pivalate which was identified by an absorption at $1780 \mathrm{~cm}^{-1}$ due to trinitrophenyl ester group. No absorption peak was found at $1810 \mathrm{~cm}^{-1}$ due to the acid chloride group. Therefore, the intermediate in this reaction was deduced as trinitrophenylester from infrared spectroscopic analysis of this model reaction.

Consequently, this active ester may react with an amino group to form the amide linkage. This reaction mechanism may also be applied to the polyamide synthesis.

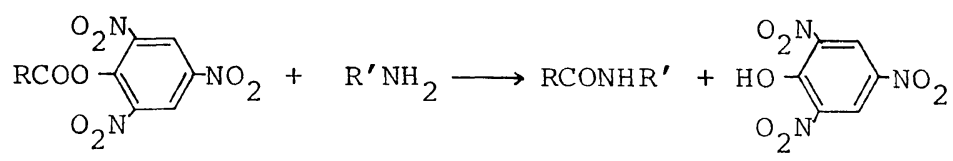

It was also reported ${ }^{9}$ that primary amine reacts secondary amine as shown below: with the pyridinium intermediate to form a

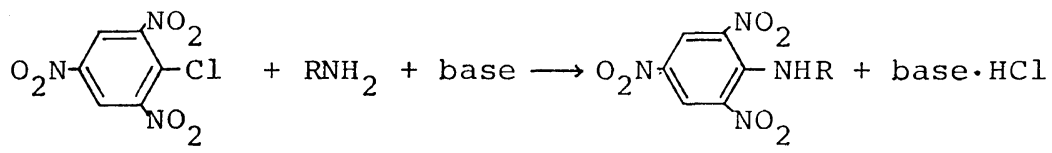




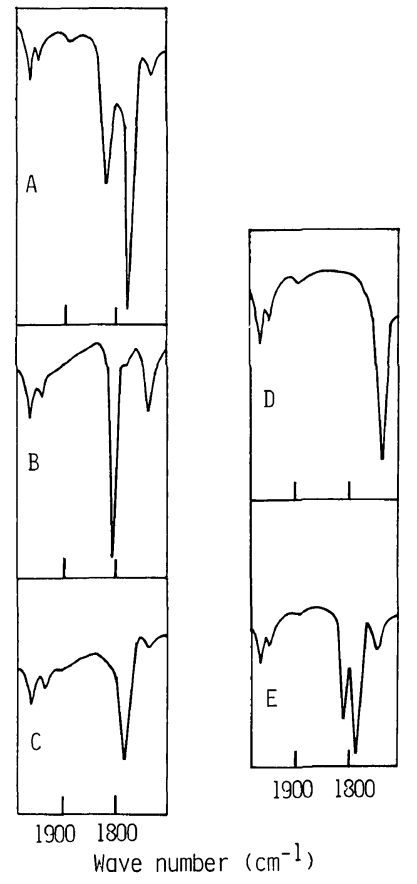

Figure 6. Infrared spectra of pivaric acid derivatives in dioxane: A, acid chloride; B, acid anhydride; C, 2,4,6trinitrophenyl ester; $\mathrm{D}$, pivaric acid; $\mathrm{E}$, reaction compound.

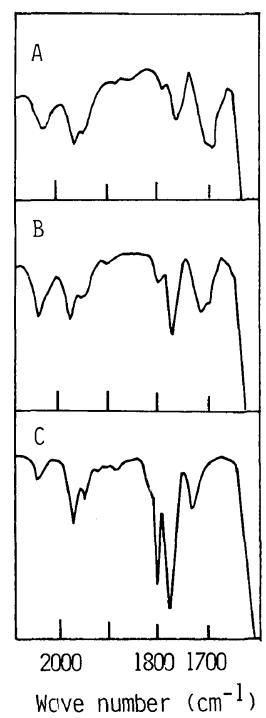

Figure 7. Infrared spectra of reaction mixtures during the reaction of picryl chloride with pivaric acid in the presence of pyridine in dioxane: A, 3 min; B, 7 min; C, 15 $\min$.
Another possible side reaction is the formation of acid anhydride as follows:
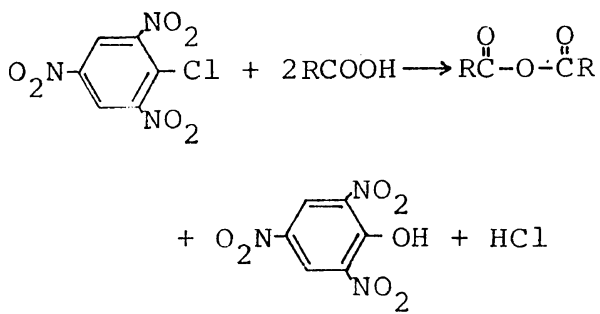

When an excess amount of picryl chloride was used in the presence of twice amount of pyridine in dioxane, absorption peaks of anhydride and trinitrophenyl ester groups could be detected by infrared spectroscopic analysis. Absorption at 1800 $\mathrm{cm}^{-1}$ due to acid anhydride groups increased with reaction time as shown in Figure 7.

The formation of acid anhydride from pivaric acid took place very rapidly in pyridine within 5 minutes. The formation of the acid anhydride seems to cause the reaction to terminate and thus prevent the growth of polymer chain in the polyamide synthesis. In order to avoid these side reactions, the pyridinium salt from picryl chloride was formed at first and then 4-ABA was added to the solution.

Table V. Effect of order of reagent addition on the polycondensation reaction

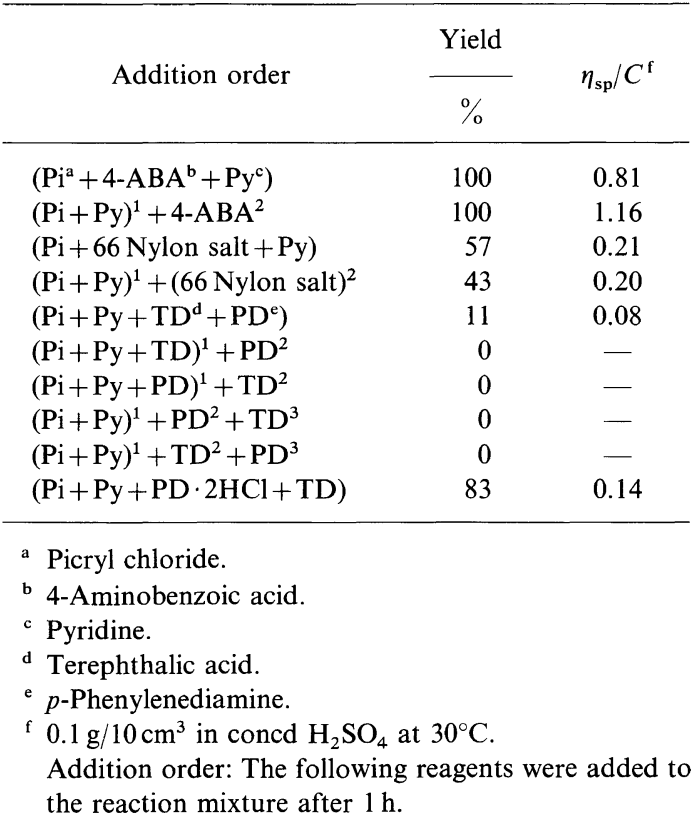


In this case a polyamide of a higher solution viscosity (1.16) was obtained compared to when the reagents were added all at once, as summarized in Table V.

On the other hand, no clear effects of the order of reagents addition were observed in case of the polycondensation of nylon 66 salt or terephthalic acid $/ p$-phenylenediamine. Polyamide from terephthalic acid and $p$-phenylenediamine was obtained in a $83 \%$ yield when $p$-phenylenediamine dihydrochloride was used. Presumably, the reactivity of both the amino and carboxylic groups may be influenced by the occurrence of side reactions (6) or (7) and other optimum reaction conditions are required in these cases.

\section{REFERENCES}

1. P. W. Morgan, "Condensation Polymers by Interfacial and Solution Methods," Interscience, New York, 1965.

2. N. Ogata, K. Sanui, and K. Iijima, J. Polym. Sci., Polym. Chem. Ed., 11, 1095 (1973).

3. N. Ogata, K. Sanui, and S. Kamiyama, J. Polym. Sci., Polym. Chem. Ed., 16, 1991 (1978).

4. N. Ogata, K. Sanui, and K. Konishi, Kobunshi Kagaku, 30, 202 (1973).

5. N. Ogata, K. Sanui, and T. Nohmi, J. Polym. Sci., Polym. Chem. Ed., 12, 1327 (1974).

6. K. Sanui, S. Tanaka, and N. Ogata, J. P'olym. Sci., Polym. Chem. Ed., 15, 1107 (1977).

7. H. Tanaka, G. Wu, Y. Iwanaga, K. Sanui, and N. Ogata, Polym. J., 14, 331 (1982).

8. R. Wittmann, Chem. Ber., 96, 771 (1963).

9. H. Kotake, K. Inomata, H. Kinoshita, K. Tanabe, and O. Miyano, Chem. Lett., 647 (1977). 